\title{
Numerical and Analytical Investigation for Darcy-Forchheimer Flow of a Williamson Fluid over a Riga Plate with Double Stratification and Cattaneo-Christov Dual Flux
}

\author{
S. Eswaramoorthi $\mathbb{D}^{1},{ }^{1}$ Nazek Alessa $\mathbb{D}^{\mathbb{D}},{ }^{2}$ M. Sangeethavaanee $\mathbb{D}^{1},{ }^{1}$ and Ngawang Namgyel $\mathbb{D}^{3}$ \\ ${ }^{1}$ Department of Mathematics, Dr. N.G.P. Arts and Science College, Coimbatore, Tamil Nadu, India \\ ${ }^{2}$ Department of Mathematical Sciences, Faculty of Science, Princess Nourah Bint Abdulrahman University, Riyadh, Saudi Arabia \\ ${ }^{3}$ Department of Humanities and Management, Jigme Namgyel Engineering College, Royal University of Bhutan, Dewathang, Bhutan
}

Correspondence should be addressed to S. Eswaramoorthi; eswaran.bharathiar@gmail.com

and Ngawang Namgyel; ngawangnamgyel@jnec.edu.bt

Received 26 May 2021; Revised 22 June 2021; Accepted 14 July 2021; Published 3 August 2021

Academic Editor: Mustafa Inc

Copyright (c) 2021 S. Eswaramoorthi et al. This is an open access article distributed under the Creative Commons Attribution License, which permits unrestricted use, distribution, and reproduction in any medium, provided the original work is properly cited.

\begin{abstract}
The Darcy-Forchheimer flow of a Williamson fluid over a Riga plate was analyzed in this paper. Energy and mass equations are modeled with Cattaneo-Christov theory and double stratifications. The governing PDE models are altered into ODE models. These models are numerically solved by MATLAB bvp4c and analytically solved by the homotopy analysis method. The impact of governing flow parameters on fluid velocity, fluid temperature, fluid concentration, skin-friction coefficient, local Nusselt number, and local Sherwood number is scrutinized via graphs and tables. We acknowledged that the speed of the fluid becomes diminishes for more presence of porosity parameter. Also, we noted that the thermal and solutal boundary layer thicknesses are waning due to their corresponding stratification parameters. In addition, the maximum decreasing percentage of skin friction is obtained when the suction/injection parameter varies from 0.0 to 0.4 for Williamson and viscous fluids. The maximum increasing percentage of local Nusselt number occurs when the suction/injection parameter varies from 0.4 to 0.8 for Williamson and viscous fluids.
\end{abstract}

\section{Introduction}

Non-Newtonian fluids are extensively implemented in diverse industrial processes such as petroleum drilling, drawing of plastic films, fibre spinning, and food production. The Williamson fluid model is one of the simplest non-Newtonian models to replicate the viscoelastic shear-thinning attributes, see Williamson [1]. The flow of thermally radiative Williamson fluid on a stretching sheet with chemical reaction was disclosed by Krishnamurthy et al. [2]. They proved the fluid temperature falling off due to the presence of the Williamson parameter. Khan et al. [3] demonstrated the impact of slip flow of Williamson nanofluid in a porous medium. They exposed that the surface drag force suppresses due to rising the Williamson fluid parameter. The $2 \mathrm{D}$ unsteady radiative Williamson fluid flow on a permeable stretching surface was deliberated by Hayat et al. [4]. They noticed that the fluid speed becomes slow when the Williamson parameter is high. Nadeem et al. [5] examined the Williamson fluid flow past a stretching sheet, and they found that the skin friction coefficient decreases with enhancing the Williamson parameter. Make use of the Keller box procedure to solve the problem of MHD flow of Williamson fluid over a stretching sheet by Salahuddin et al. [6]. Their outcome shows that the Williamson fluid parameter leads to suppress the fluid velocity. Few significant analysis for this area is seen in Refs. [7, 8]. 
Fluid flow over a porous medium is confronted in plentiful applications in industry. Few applications are wood drying, nuclear waste storage, food processing, oil purifying, drainage, and irrigation. Darcy's principle is applied to analyze the flow behavior under the condition of small velocity and low porosity. When the quantity of Reynolds number overcomes unity, the Darcy principle was not applicable. Forchheimer [9] defeated this limitation by inserting the square velocity term in the momentum equation. After that, this is known as the Forchheimer number, which is applicable for working higher Reynolds number. Numerical analysis for a Darcy-Forchheimer flow of viscous fluid over a plate was inspected by Mukhopadhyay et al. [10]. They noted that the permeability parameter leads to a decrease in the warmth of the fluid. Hayat et al. [11] demonstrate the 3D Williamson nanomaterial flow on a Darcy-Forchheimer porous medium. They concluded that the surface shear stress diminishes for growing the Forchheimer number. The Darcy-Forchheimer flow of a viscous fluid with heterogeneous-homogeneous chemical reactions was portrayed by Khan et al. [12]. Their results clearly show that the fluid speed becomes slowdown due to the availability of Darcy number. Haider et al. [13] scrutinize the Darcy-Forchheimer and slip flow of hybrid nanofluid on a rotating disk. They proved that the larger estimation of Forchheimer enhances the fluid temperature. Steady 3D Darcy-Forchheimer flow of carbon nanotubes on a rotating disk was revealed by Sadiq et al. [14]. Some important studies for these concepts are collected in Refs. [15-18].

The magnetic field plays a significant role in the development of fluid thermophysical traits. The demeanour of broadly used fluids like liquid metals, plasma, and electrolytes has a low conductor of electricity. Therefore, an external agent is required to boost up the heat transfer attributes through superior conductivity and thermophysical traits. A magnetic bar with permanently fixed magnets and alternate electronics, known as a Riga plate, can be acted as an external agent to improve fluid electricity. This plate was introduced by Gailitis and Lielausis [19]. Nanofluid flow over a Riga plate was deliberated by Ahmad et al. [20]. Nazeer et al. [21] inspected the chemically reacting Eyring-Powell nanofluid on a Riga plate. They proved that the fluid speed enhances when enhancing the modified Hartmann number. Chemically reacting Prandtl fluid on a Riga plate was addressed by Gireesha et al. [22]. Their results show that the velocity boundary layer thickens due to the more presence of the modified Hartmann number. Mehmood et al. [23] performed the impact of Soret and Dufour effects of a Casson fluid flow on a Riga plate with chemical reaction. Ayub et al. [24], Nayak et al. [25], and Rasool et al. [26] are few essential studies of fluid flow over a Riga plate.

Stratification is a natural process that combines two or more fluids with different densities, temperatures, and concentrations. The double stratification occurs due to both the heat and mass transfer differences. Cheng [27] examined the mass and heat transfer analysis of a power-law fluid in a stratified medium. He noticed that the heat transfer gradient declines for escalating the thermal stratification parameter. The radiative flow of a hyperbolic tangent fluid with chemical reaction and dual stratification's in a porous medium was elucidated by Khan et al. [28]. They found that the fluid concentration downturns for the high magnitude of the stratified thermal parameter. Rehman et al. [29] evaluated the problem of a chemically reacting Williamson fluid with dual stratification, and they have seen that the rate of heat transfer rate is declined for the presence of thermal stratification parameter. The impact of solutal and thermal stratification of a Williamson nanofluid was deliberated by Khan et al. [30]. It is noticed that the horizontal velocity suppresses when the higher magnitude of the thermal stratification parameter. Mallawi et al. [31] derived the series solution of thermally radiative non-Newtonian fluid flow with double stratifications. They have seen that the fluid concentration depresses for enhancing the solutal stratification parameter. Time-dependent MHD nanofluid flow with dual stratifications was performed by Hayat et al. [32]. They proved that surface shear stress enriches for higher values of thermal and solutal stratification parameters.

The aforementioned inspection announces that most of the researchers are involved in revealing the nature of the Darcy-Forchheimer flow with Cattaneo-Christov theory through prescribed wall temperature but not analyzed dual stratifications on a Riga plate. Therefore, our key argument is to fulfill this gap. So, our study elucidates the outcome of the Darcy-Forchheimer flow of a Williamson fluid in the presence of double stratifications, thermal radiation, and chemical reaction on a Riga plate. These types of outcomes will be definitely helpful for a thermal engineer to modeling the thermal systems. Here, the heat and mass transfer phenomena are illustrated by the Cattaneo-Christov dual flux model and the Riga plate is used to control the fluid flow.

\section{Mathematical Formulation}

Let us consider the 2D Darcy-Forchheimer flow of a Williamson fluid on a Riga plate. Here, the surface temperature and the concentration are denoted by $T_{w}$ and $C_{w}$ which are always larger than the free stream temperature $T_{\infty}$ and the free stream concentration $C_{\infty}$, respectively, see Figures 1(a) and 1(b). The thermal radiation and first-order chemical reaction are taking into account. Flow situation is manifested with double stratifications. The fluid phase is heat consumption/generation. In addition, the heat and mass transfer phenomenon is inspected through CattaneoChristov dual models. The governing equations are modeled as follows:

$$
\begin{gathered}
\frac{\partial u}{\partial x}+\frac{\partial v}{\partial y}=0 \\
u \frac{\partial u}{\partial x}+v \frac{\partial u}{\partial y}=v \frac{\partial^{2} u}{\partial y^{2}}+\sqrt{2} \Gamma v \frac{\partial u}{\partial y} \frac{\partial^{2} u}{\partial y^{2}}-\frac{v}{k_{2}} u-\frac{C_{b}}{x \sqrt{k_{2}}} u^{2} \\
+\frac{\pi J_{0} M_{0}}{8 \rho} \exp \left(-\frac{\pi}{a_{1}} y\right)
\end{gathered}
$$




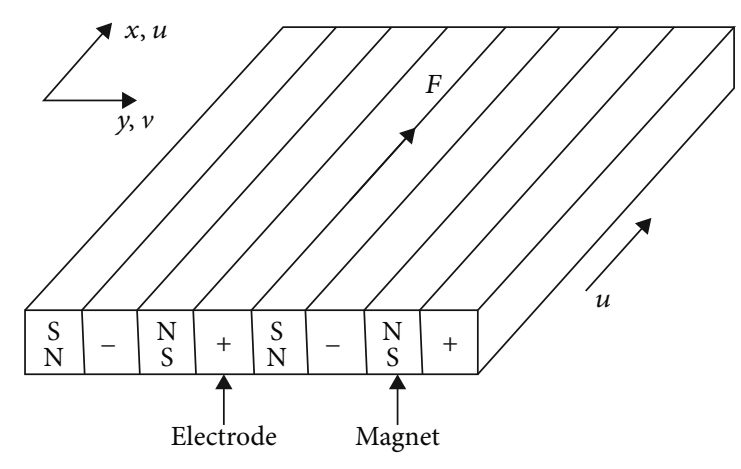

(a)

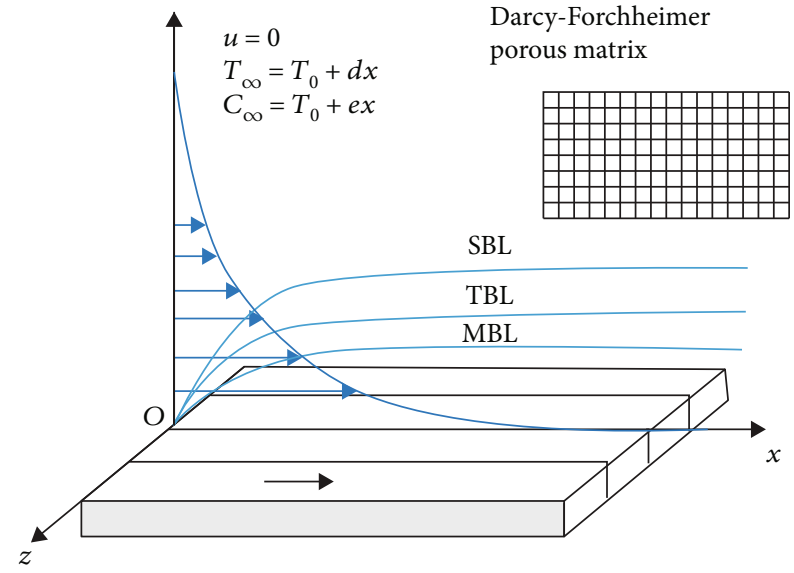

(b)

FIgURE 1: Sketch of Riga plate (a) and physical configuration of the problem (b).

$$
\begin{aligned}
u \frac{\partial T}{\partial x}+v \frac{\partial T}{\partial y}+\lambda_{T} \Omega_{T}= & \alpha \frac{\partial^{2} T}{\partial y^{2}}+\frac{1}{\rho C_{p}} \frac{16 \sigma^{*} T_{\infty}^{3}}{3 k^{*}} \frac{\partial^{2} T}{\partial y^{2}} \\
& +\frac{Q}{\rho C_{p}}\left(T-T_{\infty}\right),
\end{aligned}
$$

$$
u \frac{\partial C}{\partial x}+v \frac{\partial C}{\partial y}+\lambda_{C} \Omega_{C}=D_{B} \frac{\partial^{2} C}{\partial y^{2}}-k_{1}\left(C-C_{\infty}\right),
$$

where

$$
\begin{aligned}
\Omega_{T}= & u \frac{\partial u}{\partial x} \frac{\partial T}{\partial x}+v \frac{\partial v}{\partial y} \frac{\partial T}{\partial y}+u^{2} \frac{\partial^{2} T}{\partial x^{2}}+v^{2} \frac{\partial^{2} T}{\partial y^{2}}+2 u v \frac{\partial^{2} T}{\partial x \partial y} \\
& +u \frac{\partial v}{\partial x} \frac{\partial T}{\partial y}+v \frac{\partial u}{\partial y} \frac{\partial T}{\partial x}, \\
\Omega_{C}= & u \frac{\partial u}{\partial x} \frac{\partial C}{\partial x}+v \frac{\partial v}{\partial y} \frac{\partial C}{\partial y}+u^{2} \frac{\partial^{2} C}{\partial x^{2}}+v^{2} \frac{\partial^{2} C}{\partial y^{2}}+2 u v \frac{\partial^{2} C}{\partial x \partial y} \\
& +u \frac{\partial v}{\partial x} \frac{\partial C}{\partial y}+v \frac{\partial u}{\partial y} \frac{\partial C}{\partial x},
\end{aligned}
$$

where $u, v$ is the velocity in $x$ and $y$ directions, $v$ is the kinematic viscosity, $\Gamma$ is the time constant, $J_{0}$ is the current density, $\rho$ is the density of the fluid, $M_{0}$ is the magnetization of the magnet, $a_{1}$ is the width of the magnet and the electrodes, $C_{b}$ is the drag coefficient, $k_{2}$ is the permeability of porous medium, $T$ is the fluid temperature, $\lambda_{T}$ is the relaxation time of heat flux, $\alpha$ is the thermal diffusivity, $C_{p}$ is the specific heat, $\sigma^{*}$ is the Stefan-Boltzmann constant, $k^{*}$ is the mean absorption coefficient, $Q$ is the heat generation/absorption coefficient, $C$ is the fluid concentration, $\lambda_{C}$ is the relaxation time of mass flux, $D_{B}$ is the mass diffusivity, and $k_{1}$ is the chemical reaction parameter.
The boundary conditions are

$$
\begin{aligned}
& u=U_{w}(x), v=-V_{w}(x), T=T_{w}=T_{0}+b x, C=C_{w}=C_{0}+c x, \text { at } y=0, \\
& u \longrightarrow 0, \frac{\partial u}{\partial y} \longrightarrow 0, T \longrightarrow T_{\infty}=T_{0}+d x, C \longrightarrow C_{\infty}=C_{0}+\text { ex at } y \longrightarrow \infty
\end{aligned}
$$

Now, we consider the following dimensionless variables:

$$
\begin{gathered}
\eta=y \sqrt{\frac{a}{v},} \\
u=x a f^{\prime}, \\
v=-\sqrt{v a} f, \\
\theta(\eta)=\frac{T-T_{\infty}}{T_{w}-T_{0}}, \\
\phi(\eta)=\frac{C-C_{\infty}}{C_{w}-C_{0}} .
\end{gathered}
$$

By using (7), we can modify equations (2)-(4) as follows:

$$
\begin{aligned}
& f^{\prime \prime \prime}-f^{\prime 2}+f f^{\prime \prime}+\mathrm{We} f^{\prime \prime} f^{\prime \prime \prime}-\lambda f^{\prime}-\operatorname{Fr} f^{\prime 2}+\operatorname{Ha} e^{-\beta \eta}=0, \\
& \frac{1}{\operatorname{Pr}}\left(1+\frac{4}{3} R\right) \theta^{\prime \prime}+\operatorname{Hg} \theta+f \theta^{\prime}-f^{\prime} \theta-S_{1} f^{\prime}-\Gamma_{1}\left[f^{\prime 2} \theta\right. \\
& \left.\quad+S_{1} f^{\prime 2}-f f^{\prime} \theta^{\prime}-f f^{\prime \prime} \theta-S_{1} f f^{\prime \prime}+f^{2} \theta^{\prime \prime}\right]=0, \\
& \frac{1}{\mathrm{Sc}} \phi^{\prime \prime}-f^{\prime} \phi-S_{2} f^{\prime}+f \phi^{\prime}-\operatorname{Cr} \phi \\
& -\Gamma_{2}\left[f^{\prime 2} \phi+S_{2} f^{\prime 2}-f f^{\prime} \phi^{\prime}-f f^{\prime \prime} \phi-S_{2} f f^{\prime \prime}+f^{2} \phi^{\prime \prime}\right]=0 .
\end{aligned}
$$

$\mathrm{We}=\Gamma x \sqrt{2 a^{3} / v}$ is the Weissenberg number, $\mathrm{Ha}=\pi J_{0}$ $M_{0} / 8 \rho a^{2} x$ is the modified Hartmann number, $\beta=\pi /\left(a_{1}\right.$ $\left.(a / v)^{1 / 2}\right)$ is the dimensionless parameter, $\lambda=v / k_{2} a$ is the local porosity parameter, $\mathrm{Fr}=C_{b} / k_{2}^{1 / 2}$ is the Forchheimer number, $\operatorname{Pr}=v / a$ is the Prandtl number, $R=4 \sigma^{*} T_{\infty}^{3} / k k^{*}$ is 
the radiation parameter, $S_{1}=d / b$ is the thermal stratification parameter, $\mathrm{Hg}=Q / \rho C_{p} a$ is the heat absorption/generation parameter, $\Gamma_{1}=\lambda_{T} a$ is the heat relaxation time parameter, $\mathrm{Sc}=v / D_{B}$ is the Schmidt number, $\mathrm{Cr}=k_{1} / a$ is the chemical reaction parameter, $S_{2}=e / c$ is the solutal stratification parameter, $\Gamma_{2}=\lambda_{C} a$ is the mass relaxation time parameter, and $\mathrm{fw}=-V_{w} / \sqrt{a v}$ is the suction/injection parameter.

The corresponding boundary conditions are

$$
\begin{aligned}
f(0) & =\mathrm{fw}, \\
f^{\prime}(0) & =1, \\
\theta(0) & =1-S_{1}, \\
\phi(0) & =1-S_{2}, \\
f^{\prime}(\infty) & =0, \\
\theta(\infty) & =0, \\
\phi(\infty) & =0 .
\end{aligned}
$$

The skin friction coefficient, local Nusselt number, and local Sherwood number are expressed as

$$
\begin{aligned}
\mathrm{Cf} & =\frac{2 \tau_{w}}{\rho U_{w}^{2}} ; \\
\mathrm{Nu} & =\frac{x q_{w}}{k\left(T_{w}-T_{\infty}\right)} ; \\
\mathrm{Sh} & =\frac{x j_{w}}{D_{B}\left(C_{w}-C_{\infty}\right)} ;
\end{aligned}
$$

here, the wall shear stress, heat, and mass flux are as follows:

$$
\begin{aligned}
& \tau_{w}=\mu\left(\frac{\partial u}{\partial y}\left[1+\Gamma \sqrt{\frac{1}{2}} \frac{\partial u}{\partial y}\right]\right) ; \\
& q_{w}=-k \frac{\partial T}{\partial y}+q_{r} \\
& j_{w}=-D_{B} \frac{\partial C}{\partial y}
\end{aligned}
$$

The dimensionless form of the above parameters are expressed as

$$
\begin{aligned}
\frac{1}{2} \mathrm{Cf} \sqrt{\mathrm{Re}} & =f^{\prime \prime}(0)+\frac{\mathrm{We}}{2} f^{\prime \prime}(0)^{2} ; \\
\frac{\mathrm{Nu}}{\sqrt{\mathrm{Re}}} & =-\left(1+\frac{4}{3} R\right) \theta^{\prime}(0) ; \\
\frac{\mathrm{Sh}}{\sqrt{\mathrm{Re}}} & =-\phi^{\prime}(0) .
\end{aligned}
$$

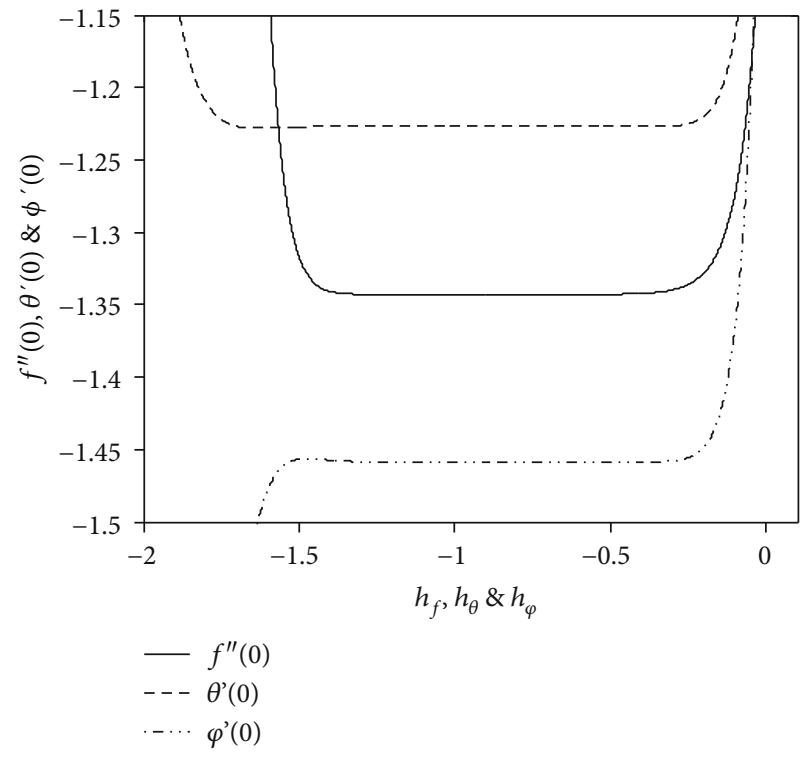

Figure $2: h$ - curve with $18^{\text {th }}$ order of approximation.

TABLE 1: Order of order approximations and CPU timing.

\begin{tabular}{lcccc}
\hline Order & $-f^{\prime \prime}(0)$ & $-\theta^{\prime}(0)$ & $-\phi^{\prime}(0)$ & CPU timings $(\mathrm{sec})$. \\
\hline 1 & 1.27000 & 1.21054 & 1.43054 & 0.58 \\
5 & 134103 & 1.22687 & 1.45881 & 4.73 \\
10 & 1.34290 & 1.22692 & 1.45860 & 33.06 \\
13 & 1.34294 & 1.22692 & 1.45860 & 47.64 \\
15 & 1.34294 & 1.22692 & 1.45860 & 139.76 \\
20 & 1.34294 & 1.22692 & 1.45860 & 646.61 \\
25 & 1.34294 & 1.22692 & 1.45860 & 2376.22 \\
30 & 1.34294 & 1.22692 & 1.45860 & 7157.92 \\
\hline
\end{tabular}

\section{Solutions}

3.1. Numerical Solution. In this section, the bvp4c solver has been used for gaining the solution. In order to solve the problem, equations (8)-(10) are commuted into a system of firstorder differential equations with the boundary conditions also modified in the same manner. For this, let us take

$$
\begin{aligned}
f & =y_{1}, \\
f^{\prime} & =y_{2}, \\
f^{\prime \prime} & =y_{3}, \\
\theta & =y_{4}, \\
\theta^{\prime} & =y_{5}, \\
\phi & =y_{6}, \\
\phi^{\prime} & =y_{7} .
\end{aligned}
$$


TABLe 2: Numerical and analytical computation of local skin friction coefficient, local Nusselt number, and local Sherwood number for various values of $\mathrm{We}, \lambda$, Fr, fw, and $\mathrm{Ha}$.

\begin{tabular}{|c|c|c|c|c|c|c|c|c|c|c|}
\hline \multirow{2}{*}{ We } & \multirow{2}{*}{$\lambda$} & \multirow{2}{*}{$\mathrm{Fr}$} & \multirow{2}{*}{ fw } & \multirow{2}{*}{$\mathrm{Ha}$} & \multicolumn{2}{|c|}{ Skin friction coefficient } & \multicolumn{2}{|c|}{ Local Nusselt number } & \multicolumn{2}{|c|}{ Local Sherwood number } \\
\hline & & & & & Numerical & Analytical & Numerical & Analytical & Numerical & Analytical \\
\hline 0.0 & 0.2 & 0.4 & 0.3 & 0.3 & -1.21627 & -1.21624 & 2.06338 & 2.06344 & 1.46840 & 1.46841 \\
\hline 0.1 & & & & & -1.19072 & -1.19070 & 2.05471 & 2.05477 & 1.46383 & 1.46385 \\
\hline 0.2 & & & & & -1.16263 & -1.16259 & 2.04481 & 2.04487 & 1.45858 & 1.45860 \\
\hline 0.3 & & & & & -1.13089 & -1.13083 & 2.03314 & 2.03319 & 1.45233 & 1.45235 \\
\hline 0.4 & & & & & -1.09319 & -1.09164 & 2.01848 & 2.01859 & 1.44438 & 1.44442 \\
\hline 0.5 & & & & & -0.99995 & -0.99998 & 1.99720 & 1.99782 & 1.43257 & 1.43294 \\
\hline \multirow[t]{6}{*}{0.2} & 0.0 & 0.4 & 0.3 & 0.3 & -1.07827 & -1.07818 & 2.07408 & 2.07422 & 1.47298 & 1.47303 \\
\hline & 0.1 & & & & -1.12135 & -1.12130 & 2.05904 & 2.05913 & 1.46558 & 1.46561 \\
\hline & 0.2 & & & & -1.16263 & -1.16259 & 2.04481 & 2.04487 & 1.45858 & 1.45860 \\
\hline & 0.3 & & & & -1.20228 & -1.20225 & 2.03132 & 2.03136 & 1.45194 & 1.45195 \\
\hline & 0.4 & & & & -1.24046 & -1.24042 & 2.01851 & 2.01853 & 1.44563 & 1.44564 \\
\hline & 0.5 & & & & -1.27728 & -1.27725 & 2.00630 & 2.00634 & 1.43961 & 1.43964 \\
\hline \multirow[t]{5}{*}{0.2} & 0.2 & 0.0 & 0.3 & 0.3 & -1.05656 & -1.05652 & 2.06805 & 2.06811 & 1.47074 & 1.47076 \\
\hline & & 0.3 & & & -1.13706 & -1.13702 & 2.05038 & 2.05043 & 1.46149 & 1.46151 \\
\hline & & 0.5 & & & -1.18763 & -1.18758 & 2.03940 & 2.03945 & 1.45575 & 1.45576 \\
\hline & & 0.8 & & & -1.25946 & -1.25942 & 2.02397 & 2.02406 & 1.44768 & 1.44773 \\
\hline & & 1.0 & & & -1.30495 & -1.30544 & 2.01430 & 2.01498 & 1.44262 & 1.44322 \\
\hline \multirow[t]{5}{*}{0.2} & 0.2 & 0.4 & -1.0 & 0.3 & -0.68939 & -0.68936 & 1.22935 & 1.22940 & 0.91349 & 0.91350 \\
\hline & & & -0.5 & & -0.83580 & -0.83579 & 1.47333 & 1.47339 & 1.07673 & 1.07675 \\
\hline & & & 0.0 & & -1.02546 & -1.02543 & 1.79633 & 1.79639 & 1.29221 & 1.29223 \\
\hline & & & 0.5 & & -1.26411 & -1.26408 & 2.24148 & 2.24153 & 1.59103 & 1.59105 \\
\hline & & & 1.0 & & -1.55195 & -1.55182 & 2.88826 & 2.88923 & 2.03343 & 2.03242 \\
\hline \multirow[t]{5}{*}{0.2} & 0.2 & 0.4 & 0.3 & 0.0 & -1.29871 & -1.29869 & 1.99254 & 1.99255 & 1.43332 & 1.43333 \\
\hline & & & & 0.5 & -1.07378 & -1.07374 & 2.07612 & 2.07619 & 1.47399 & 1.47401 \\
\hline & & & & 1.0 & -0.85646 & -0.85641 & 2.14556 & 2.14568 & 1.50882 & 1.50886 \\
\hline & & & & 1.5 & -0.64420 & -0.64411 & 2.20587 & 2.20586 & 1.53970 & 1.53989 \\
\hline & & & & 2.0 & -0.43568 & -0.43431 & 2.25965 & 2.26084 & 1.56771 & 1.57081 \\
\hline
\end{tabular}

The system of equations are

$$
\begin{aligned}
& y_{1}^{\prime}=y_{2}, \\
& y_{2}^{\prime}=y_{3}, \\
& y_{3}^{\prime}=\frac{y_{2}^{2}-y_{1} y_{3}-\mathrm{Ha} e^{-\beta \eta}+\lambda y_{2}+\mathrm{Fr} y_{2}^{2}}{1+\mathrm{We} y_{3}}, \\
& y_{4}^{\prime}=y_{5}, \\
& y_{5}^{\prime}=\frac{-y_{1} y_{5}+y_{2} y_{4}+S_{1} y_{2}-\operatorname{Hg} y_{4}+\Gamma_{1}\left[y_{2}^{2} y_{4}+S_{1} y_{2}^{2}-y_{1} y_{2} y_{5}-y_{1} y_{3} y_{4}-S_{1} y_{1} y_{3}\right]}{(1 / P r)(1+(4 / 3) R)-\Gamma_{1} y_{1}^{2}}, \\
& y_{6}^{\prime}=y_{7}, \\
& y_{7}^{\prime}=\frac{-y_{1} y_{7}+y_{2} y_{6}+S_{2} y_{2}+\operatorname{Cr} y_{6}+\Gamma_{2}\left[y_{2}^{2} y_{6}+S_{2} y_{2}^{2}-y_{1} y_{2} y_{7}-y_{1} y_{3} y_{6}-S_{2} y_{1} y_{3}\right]}{(1 / \mathrm{Sc})-\Gamma_{2} y_{1}^{2}} .
\end{aligned}
$$


With the boundary conditions

$$
\begin{aligned}
y_{1}(0) & =\mathrm{fw}, \\
y_{2}(0) & =1, \\
y_{2}(\infty) & =0, \\
y_{4}(0) & =1-S_{1}, \\
y_{4}(\infty) & =0, \\
y_{6}(0) & =1-S_{2}, \\
y_{6}(\infty) & =0 .
\end{aligned}
$$

The above set of equations are numerically solved by MATLAB built-in function bvp4c.

3.2. HAM Solution. The obtained ODE's (8)-(10) with conditions (11) are analytically solved by applying the HAM scheme. Because this method is powerful tool for solving nonlinear problems, see Sarwar and Rashidi [33]. Let the initial approximations are chosen as $f_{0}(\eta)=\mathrm{fw}+1-1 / e^{\eta}$, $\theta_{0}(\eta)=\left(1-S_{1}\right) / e^{\eta}$, and $\phi_{0}(\eta)=\left(1-S_{2}\right) / e^{\eta}$, and linear operators are $L_{f}=D^{3} f-D f, L_{\theta}=D^{2} \theta-\theta$, and $L_{\phi}=D^{2} \phi-\phi$, where $D$ is the differential operator and the linear property is $L_{f}\left[C_{1}+C_{2} e^{\eta}+C_{3}\left(1 / e^{\eta}\right)\right]=0=L_{\theta}\left[C_{4} e^{\eta}+C_{5}\left(1 / e^{\eta}\right)\right]=L_{\phi}\left[C_{6} e^{\eta}\right.$ $\left.+C_{7}\left(1 / e^{\eta}\right)\right]$, where $C_{k}(k=1-7)$ are constants.

After implementing the $i^{\text {th }}$ order HAM technique, we found the following:

$$
\begin{aligned}
& f_{i}(\eta)=f_{i}^{*}(\eta)+C_{1}+C_{2} e^{\eta}+C_{3} \frac{1}{e^{\eta}}, \\
& \theta_{i}(\eta)=\theta_{i}^{*}(\eta)+C_{4} e^{\eta}+C_{5} \frac{1}{e^{\eta}}, \\
& \phi_{i}(\eta)=\phi_{i}^{*}(\eta)+C_{6} e^{\eta}+C_{7} \frac{1}{e^{\eta}} .
\end{aligned}
$$

Here, $f_{i}^{*}(\eta), \theta_{i}^{*}(\eta)$, and $\phi_{i}^{*}(\eta)$ are the particular solutions.

These HAM techniques have the parameters $\left(h_{f}, h_{\theta}\right.$, and $h_{\phi}$ ), and these are responsible for the convergence of solutions, see Refs. [34-37]. Figure 2 portrays the range value of $h_{f}, h_{\theta}$, and $h_{\phi}$ are $-1.3 \leq h_{f} \leq-0.4,-1.6 \leq h_{\theta} \leq-0.25$, and $-1.4 \leq h_{\phi} \leq-0.35$. We assign $h_{f}=h_{\theta}=h_{\phi}=-0.9$ for better convergency.

\section{Results and Discussion}

Here, we revealed the results by graphs and tables which describes the shift in velocity, temperature, concentration, skin friction coefficient, local Nusselt number, and local Sherwood number concerning the disparate values of the parameters, such as Weissenberg number (We), local porosity parameter $(\lambda)$, Forchheimer number $(\mathrm{Fr})$, modified Hartmann number (Ha), thermal radiation parameter $(R)$, thermal stratification parameter $\left(S_{1}\right)$, heat generation/absorption parameter $(\mathrm{Hg})$, heat relaxation time parameter $\left(\Gamma_{1}\right)$, chemical reaction parameter $(\mathrm{Cr})$, solute stratification parameter $\left(S_{2}\right)$, mass relaxation time parameter $\left(\Gamma_{2}\right)$, and the suction/injection
TABle 3: Numerical and analytical computation of local Nusselt number for various values of $R, \mathrm{Hg}, S_{1}$, and $\Gamma_{1}$.

\begin{tabular}{cccccc}
\hline \multirow{2}{*}{$R$} & \multirow{2}{*}{$\mathrm{Hg}$} & $S_{1}$ & $\Gamma_{1}$ & \multicolumn{2}{c}{ Local Nusselt number } \\
& & & & Numerical & Analytical \\
\hline 0.0 & -0.5 & 0.2 & 0.1 & 1.72351 & 1.72355 \\
0.3 & & & & 1.92821 & 1.92826 \\
0.5 & & & & 2.04481 & 2.04487 \\
0.7 & & & & 2.15002 & 2.15009 \\
1.0 & & & & 2.29156 & 2.29164 \\
0.5 & -0.5 & 0.2 & 0.1 & 2.04481 & 2.04487 \\
& -0.2 & & & 1.88531 & 1.88544 \\
& 0.0 & & & 1.76494 & 1.94091 \\
& 0.2 & & & 1.62586 & 1.62695 \\
& 0.5 & & & 1.21530 & 1.27139 \\
0.5 & -0.5 & 0.0 & 0.1 & 2.33479 & 2.33481 \\
& & 0.2 & & 2.04481 & 2.04487 \\
& & 0.4 & & 1.75483 & 1.75492 \\
& & 0.6 & & 1.46484 & 1.46498 \\
& & 0.8 & & 1.17486 & 1.17503 \\
& & 1.0 & & 0.88488 & 0.88508 \\
0.5 & -0.5 & 0.2 & 0.0 & 1.90591 & 1.90597 \\
& & & 0.1 & 2.04481 & 2.04487 \\
& & & 0.2 & 2.18818 & 2.18824 \\
& & & 0.3 & 2.33609 & 2.33615 \\
& & & 0.4 & 2.48859 & 2.48865 \\
\hline
\end{tabular}

TABLE 4: Numerical and analytical computation of local Sherwood number for various values of $\mathrm{Cr}, \mathrm{S}_{2}$, and $\Gamma_{2}$.

\begin{tabular}{lcccc}
\hline \multirow{2}{*}{ r } & $S_{2}$ & $\Gamma_{2}$ & \multicolumn{2}{c}{ Local Sherwood number } \\
& & & Numerical & Analytical \\
\hline-0.5 & 0.2 & 0.1 & 0.90317 & 0.89525 \\
-0.2 & & & 1.06313 & 1.06391 \\
0.0 & & & 1.14498 & 1.14517 \\
1.5 & & & 1.31565 & 1.31568 \\
1.0 & & & 1.45858 & 1.45860 \\
1.0 & 0.0 & 0.1 & 1.69469 & 1.69469 \\
& 0.2 & & 1.45858 & 1.45860 \\
& 0.4 & & 1.22248 & 1.22250 \\
& 0.6 & & 0.98637 & 0.98640 \\
& 0.8 & & 0.75026 & 0.75030 \\
& 1.0 & & 0.51416 & 0.51421 \\
1.0 & 0.2 & 0.0 & 1.37297 & 1.37298 \\
& & 0.1 & 1.45858 & 1.45860 \\
& & 0.2 & 1.54716 & 1.54718 \\
& & 0.3 & 1.63881 & 1.63884 \\
& & 0.4 & 1.72399 & 1.73363 \\
\hline
\end{tabular}

parameter $(\mathrm{fw})$. The numerically obtained values are compared with the results fetched by the analytical approach by HAM. 


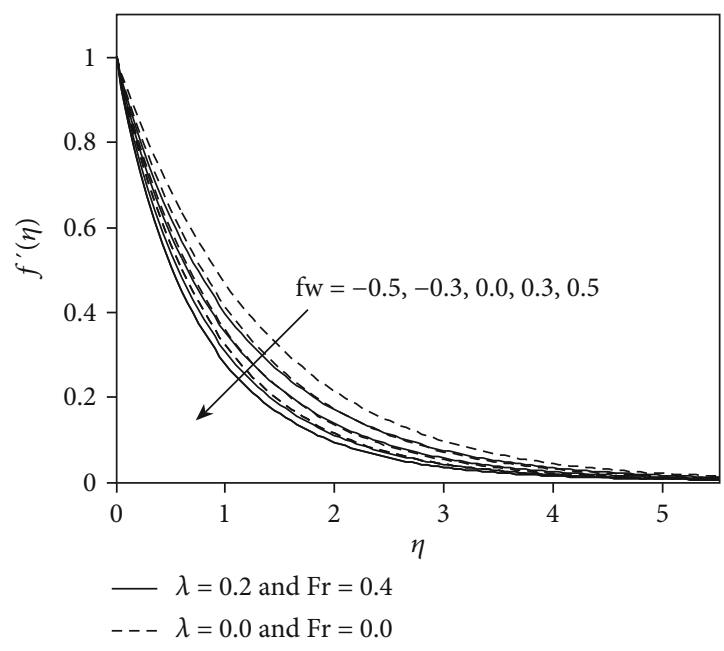

(a)

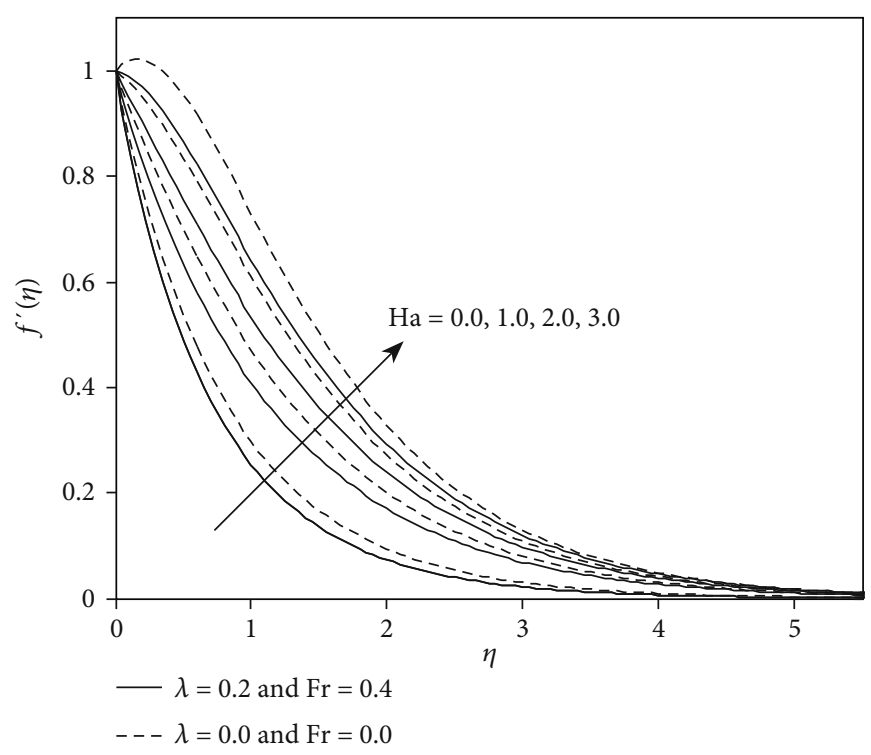

(b)

FIgURE 3: The velocity profile for disparate values of $\mathrm{fw}$ (a) and $\mathrm{Ha}(\mathrm{b})$.

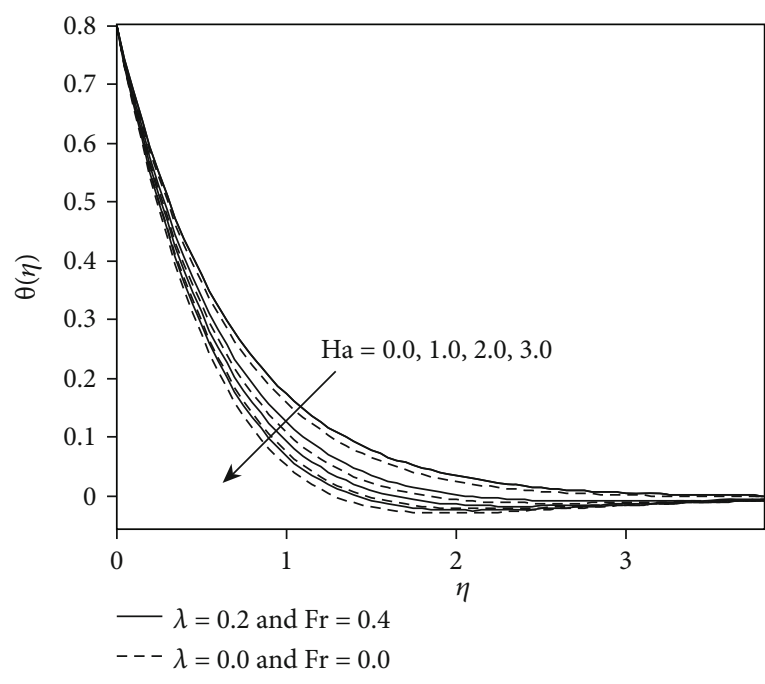

(a)

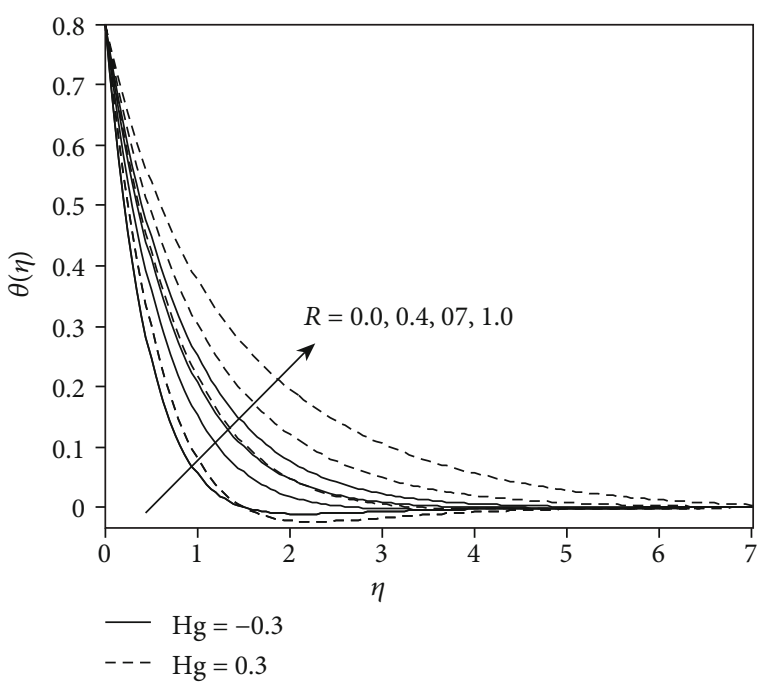

(b)

Figure 4: The temperature profile for disparate values of $\mathrm{Ha}(\mathrm{a})$ and $R$ (b).

Table 1 provides the HAM order and CPU timings. From this table, we concluded that $13^{\text {th }}$ order is sufficient for all profiles. Table 2 delineates the changes of skin friction coefficient, local Nusselt number, and local Sherwood number for the distinct values of $\mathrm{We}, \lambda, \mathrm{Fr}, \mathrm{Ha}$, and fw. We noted that the surface shear stress upsurges when heightening the We and $\mathrm{Ha}$ values and it declines for enhancing the $\lambda$, Fr, and $\mathrm{fw}$ values. The local Nusselt and Sherwood numbers reduce for raising the $\mathrm{We}, \lambda$, and $\mathrm{Fr}$, and it rises for increasing the $\mathrm{Ha}$ and $\mathrm{fw}$. Table 3 describes the influence of $R, \mathrm{Hg}, S_{1}$, and $\Gamma_{1}$ over the heat flux. The heat transfer gradient decimates when developing the $\mathrm{Hg}$ and $S_{1}$ values, and it grows when growing the $R$ and $\Gamma_{1}$ values. Table 4 helps to figure out the shift of mass flux for the various values of $\mathrm{Cr}, S_{2}$, and $\Gamma_{2}$. The mass transfer rate escalates for the enriching values of $\mathrm{Cr}$ and $\Gamma_{2}$, and it suppresses for increasing $S_{2}$ values. Also, we proved that our numerical and analytical results are almost same.

Figures 3(a) and 3(b) establish the impact of fw (a) and $\mathrm{Ha}$ (b) on velocity profile for DFRP and NDFRP. We uncovered that the fluid speed aggravates due to more presence of Hartmann number and quite the opposite behavior is obtained for the fw parameter. The MBLT is high in NDFRP compared to DFRP for both parameters. The outcomes for disparate values of $\mathrm{Ha}$ and $R$ on temperature profile are 


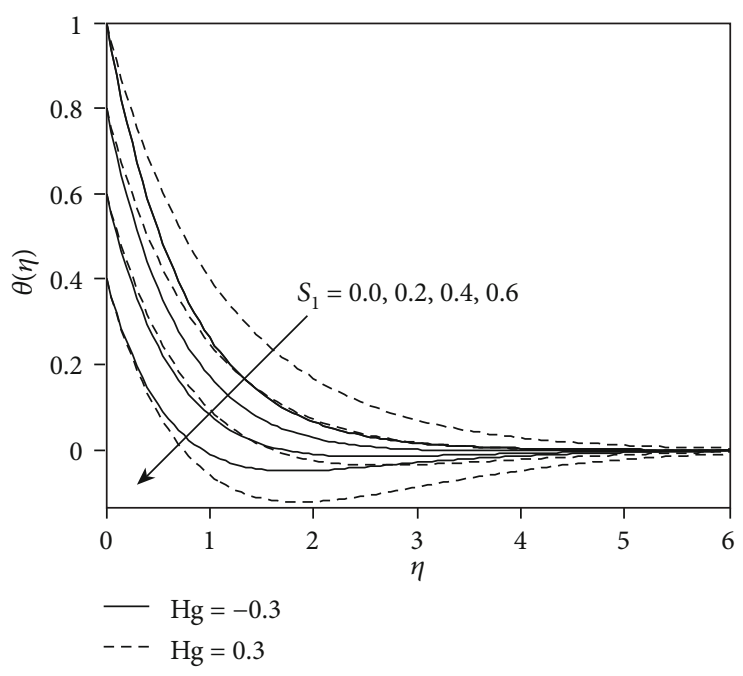

(a)

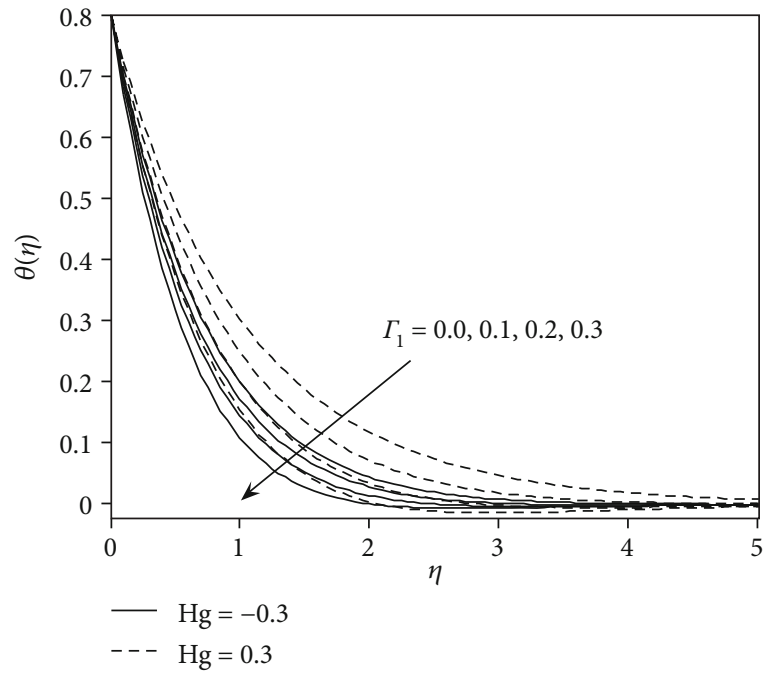

(b)

Figure 5: The temperature profile for disparate values of $S_{1}$ (a) and $\Gamma_{1}$ (b).

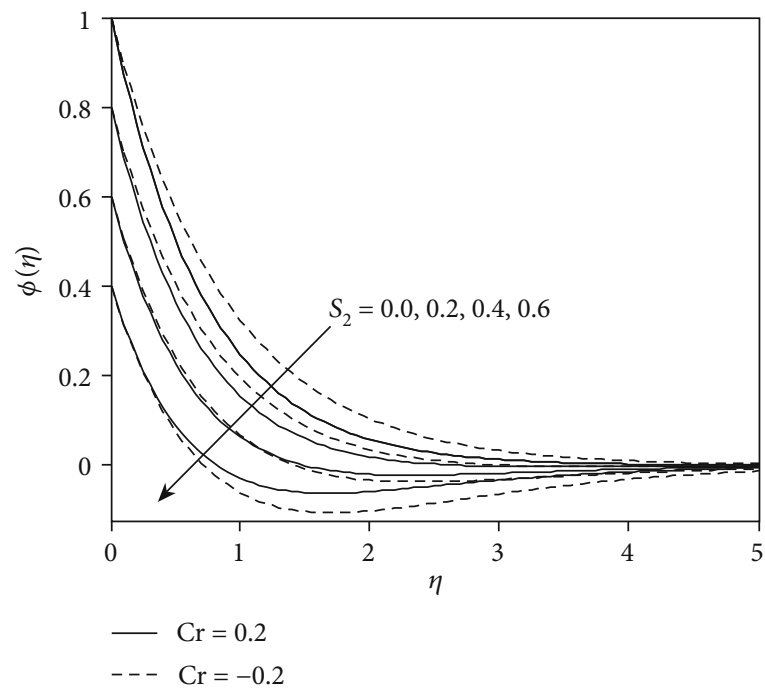

(a)

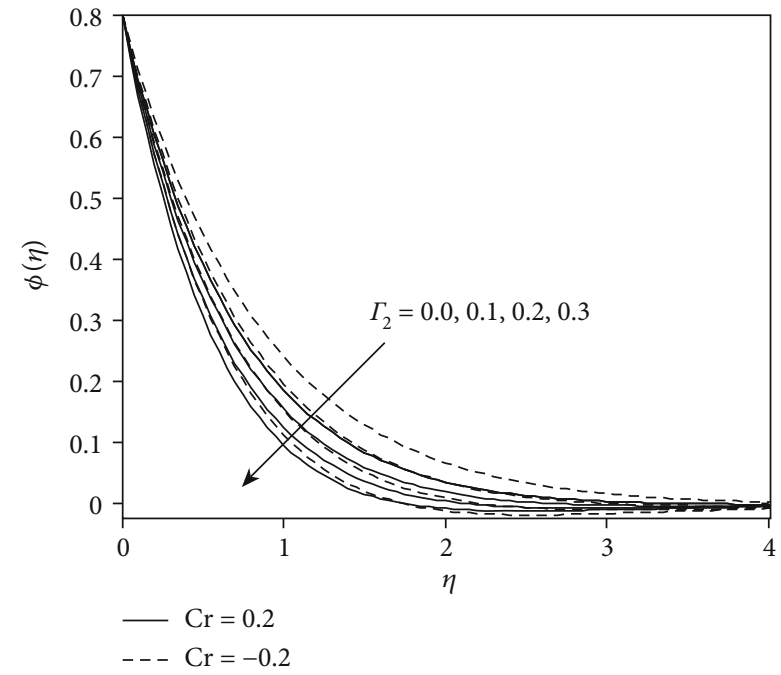

(b)

Figure 6: The concentration profile for disparate values of $S_{2}$ (a) and $\Gamma_{2}$ (b).

illustrated in Figures 4(a) and 4(b) for DFRP and NDFRP (a) and heat consumption/generation (b). We ascertained that the fluid temperature dwindles because of the high quantity of Hartmann number. However, it is enhanced for raising the radiation parameter. Figures 5(a) and 5(b) explains the changes of fluid temperature for distinct values of $S_{1}$ (a) and $\Gamma_{1}$ (b) for heat generation/consumption fluid. We revealed that the fluid warmness becomes subsides for hike values of $S_{1}$ and $\Gamma_{1}$. In addition, the thermal boundary layer thickness is large in the heat generation case compared to the heat consumption case. The significance of $S_{2}$ and $\Gamma_{2}$ on concentration profile on destructive chemical reaction and the generative chemical reaction is plotted in Figures 6(a) and 6(b). We ascertained that the fluid concentration lessens for large values of $S_{2}$ and $\Gamma_{2}$.
The variations of SFC for distinct combination of fw and $\mathrm{Ha}$ (a) for DF flow (lower plate) and NDF flow (upper plate) and (b) for DF flow of Williamson fluid (upper plate) and viscous fluid (lower plate) are portrayed in Figures 7(a) and 7(b). We found that the plate shear stress depresses for enhancing values of $\mathrm{fw}$, and it raises for rising the values of Hartmann number. Figures $8(\mathrm{a})$ and $8(\mathrm{~b})$ disclose the changes of LNN for disparate combination of $\mathrm{fw}$ and $\mathrm{Ha}$ (a) for DF flow (lower plate) and NDF flow (upper plate) and (b) for DF flow of Williamson fluid (lower plate) and viscous fluid (upper plate). We noted that heat transfer gradient escalates for increasing the values of fw and Ha. The alternations of LNN for various combination of (a) fw and $\Gamma_{1}$ with $\mathrm{Hg}=-0.5$ (upper plate) and $\mathrm{Hg}=0.5$ (lower plate) and (b) 


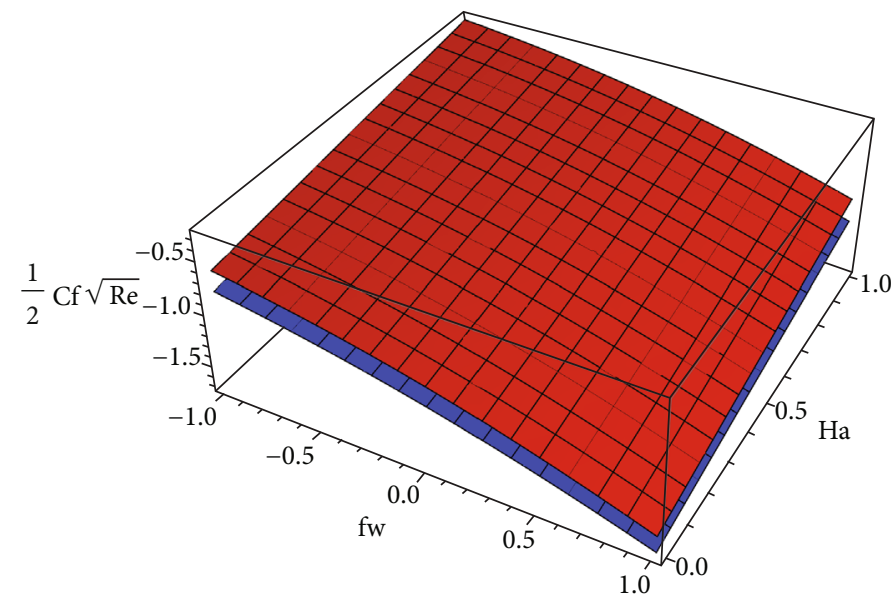

(a)

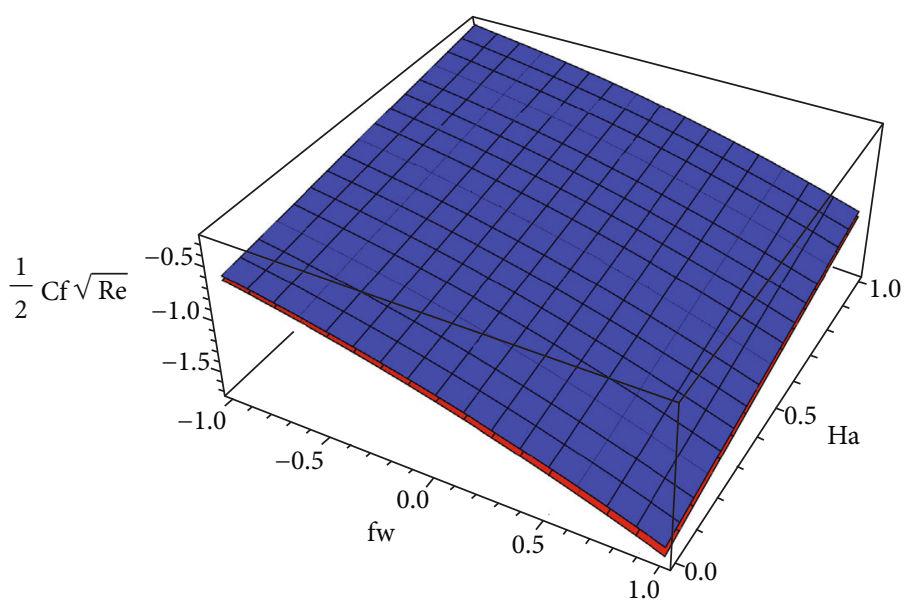

(b)

Figure 7: The variations of SFC for distinct combination of fw and Ha (a) for DF flow (lower plate) and NDF flow (upper plate) and (b) for DF flow of Williamson fluid (upper plate) and viscous fluid (lower plate).

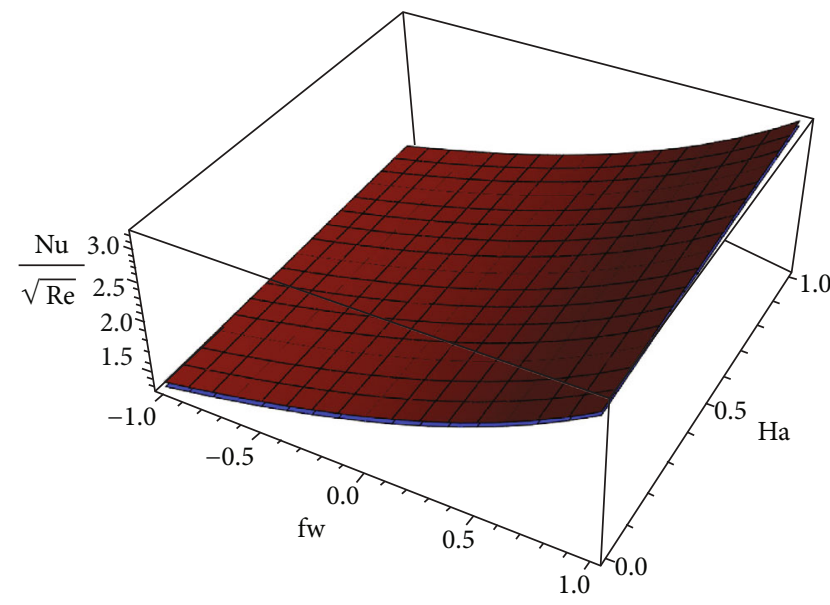

(a)

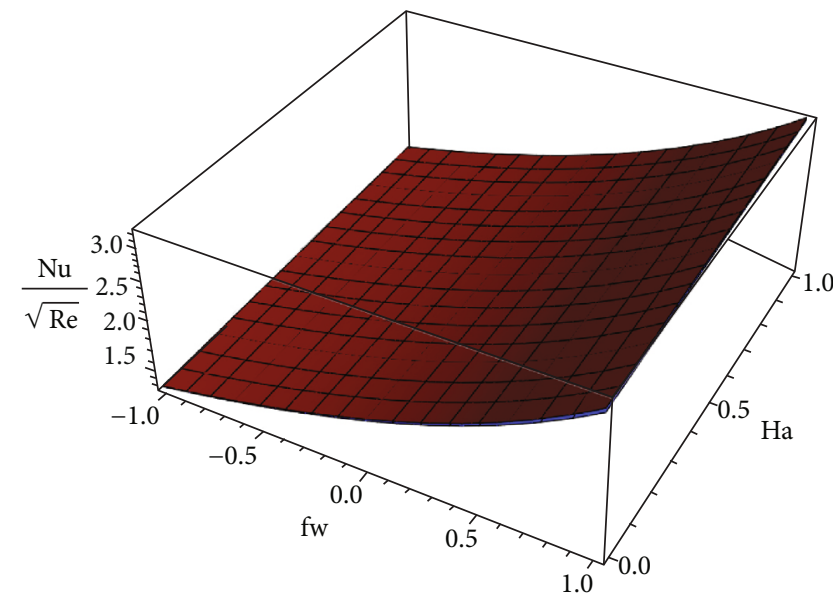

(b)

FIgURE 8: The variations of LNN for distinct combination of fw and $\mathrm{Ha}$ (a) for DF flow (lower plate) and NDF flow (upper plate) and (b) for DF flow of Williamson fluid (lower plate) and viscous fluid (upper plate). 


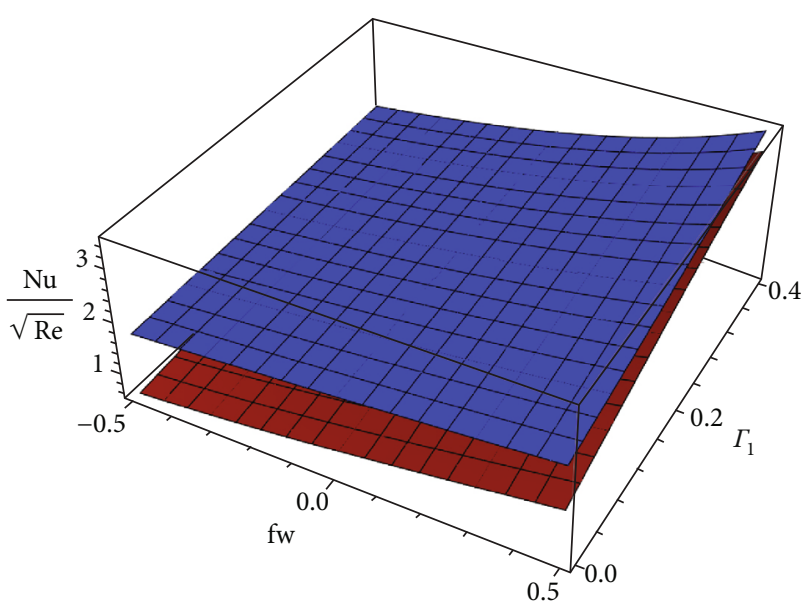

(a)

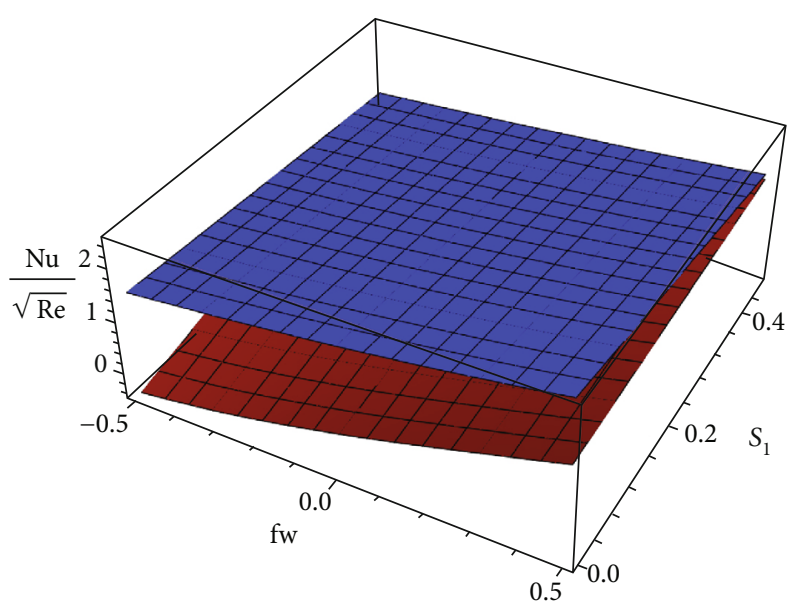

(b)

FIgURE 9: The variations of LNN for distinct combination of (a) $\mathrm{fw}$ and $\Gamma_{1}$ with $\mathrm{Hg}=-0.5$ (upper plate) and $\mathrm{Hg}=0.5$ (lower plate) and (b) fw and $S_{1}$ with $\mathrm{Hg}=-0.5$ (upper plate) and $\mathrm{Hg}=0.5$ (lower plate).

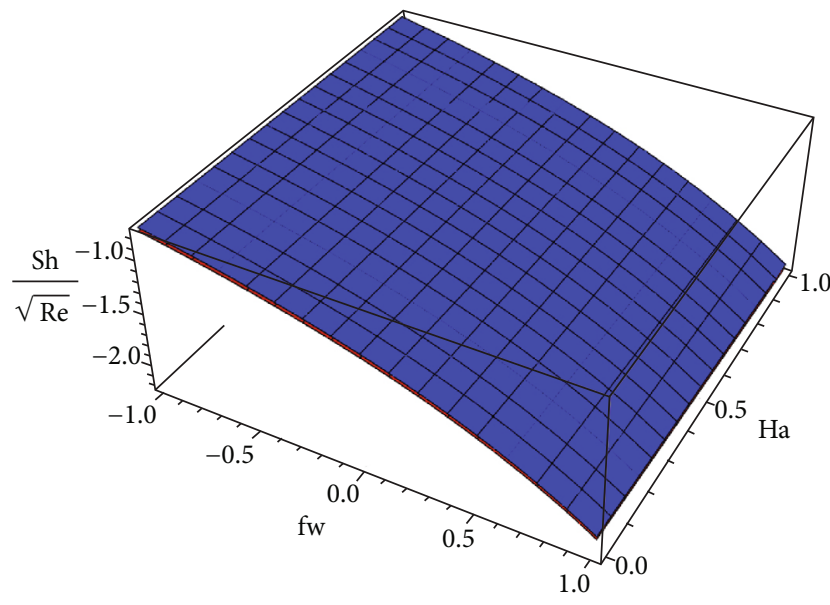

(a)

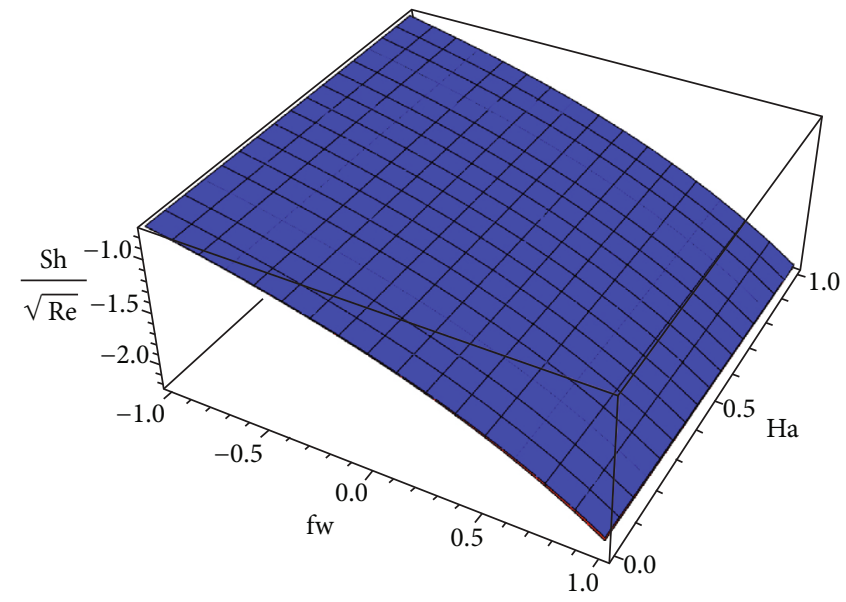

(b)

FIGURE 10: The variations of LSN for distinct combination of fw and Ha (a) for DF flow (upper plate) and NDF flow (lower plate) and (b) for DF flow of Williamson fluid (upper plate) and viscous fluid (lower plate).

fw and $S_{1}$ with $\mathrm{Hg}=-0.5$ (upper plate) and $\mathrm{Hg}=0.5$ (lower plate) are plotted in Figures 9(a) and 9(b). We noticed that the heat transfer gradient upgrades for the available of $\mathrm{fw}$, and it weakens for high quantity of $S_{1}$ and $\Gamma_{1}$. In addition, the larger heat transfer gradient occurs in heat consumption case compared to heat generation case. Figures 10(a) and 10(b) express the LSN variation with respect to the distinct combination of $\mathrm{fw}$ and $\mathrm{Ha}$ (a) for DF flow (upper plate) and NDF flow (lower plate) and (b) for DF flow of Williamson fluid (upper plate) and viscous fluid (lower plate). We proved that the mass transfer gradient slashes due to larger values of fw and Ha. The deviation of LSN for different combination of (a) fw and $\Gamma_{2}$ with generative chemical reaction (upper plate) and destructive chemical reaction (lower plate) and (b) fw and $S_{2}$ with generative chemical reaction (upper plate) and destructive chemical reaction (lower plate) is shown in Figures 11(a) and 11(b). We concluded that the LSN declines for upgrading the values of fw, $\Gamma_{2}$, and $S_{2}$.

The decrement percentage of SFC for various values of $\mathrm{fw}$ on Williamson fluid and viscous fluid is plotted in Figures 12(a) and 12(b) and observed that the maximum decreasing percentage of surface shear stress is obtained when fw varies from 0.0 to 0.4 for both fluids. Figures 13(a) and 13(b) give the increment percentage of LNN for various values of fw on Williamson fluid and viscous fluid. The maximum increment percentage has occurred when fw vary from 0.4 to 0.8 for both fluids. The decrement percentage of LSN for various values of $\mathrm{fw}$ on Williamson fluid and viscous fluid is illustrated in Figures 14(a) and 14(b), and we have seen that the maximum decrement percentage occurred when $\mathrm{fw}$ varies from 0.4 to 0.8 for both fluids. Figures 15(a) and 15(b) display the decrement/increment percentage of LNN 


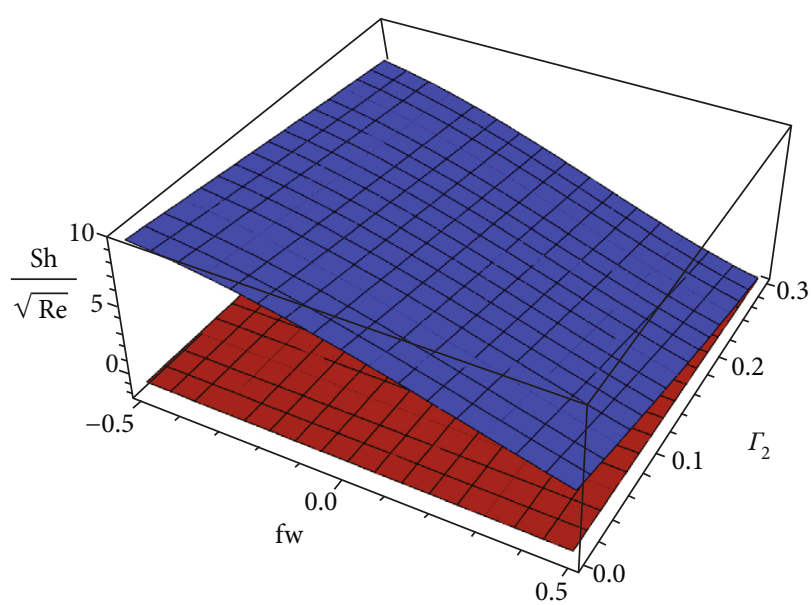

(a)

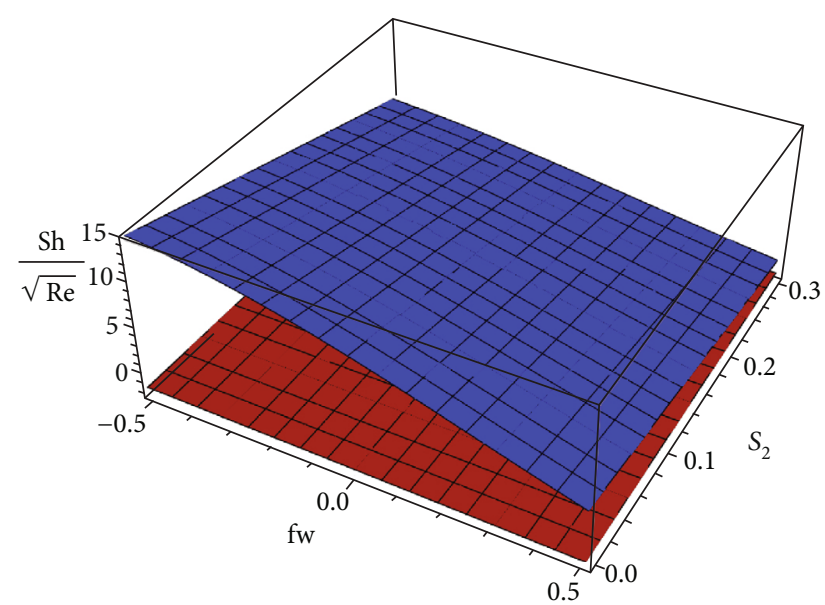

(b)

FIgURE 11: The variations of LSN for distinct combination of (a) fw and $\Gamma_{2}$ with generative chemical reaction (upper plate) and destructive chemical reaction (lower plate) and (b) fw and $S_{2}$ with generative chemical reaction (upper plate) and destructive chemical reaction (lower plate).

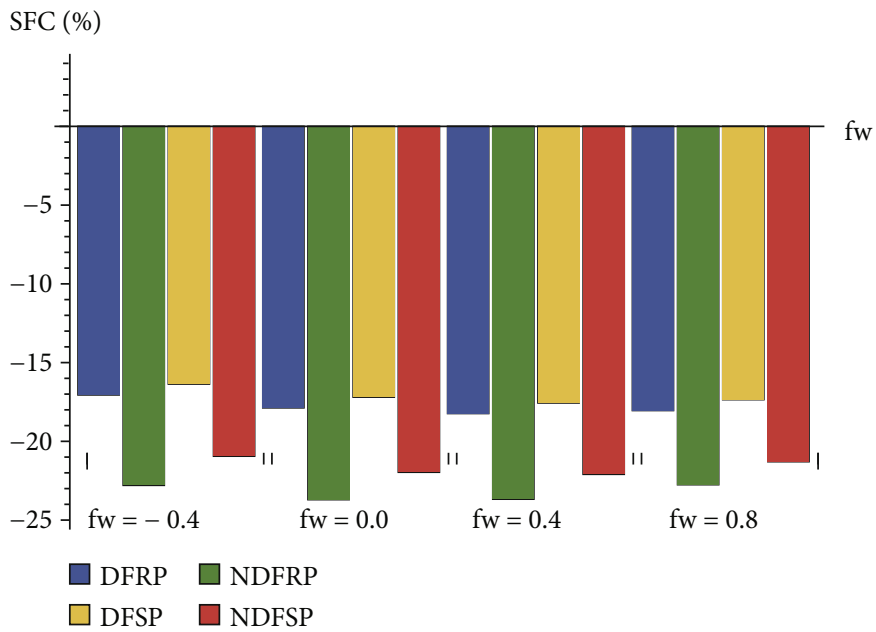

(a)

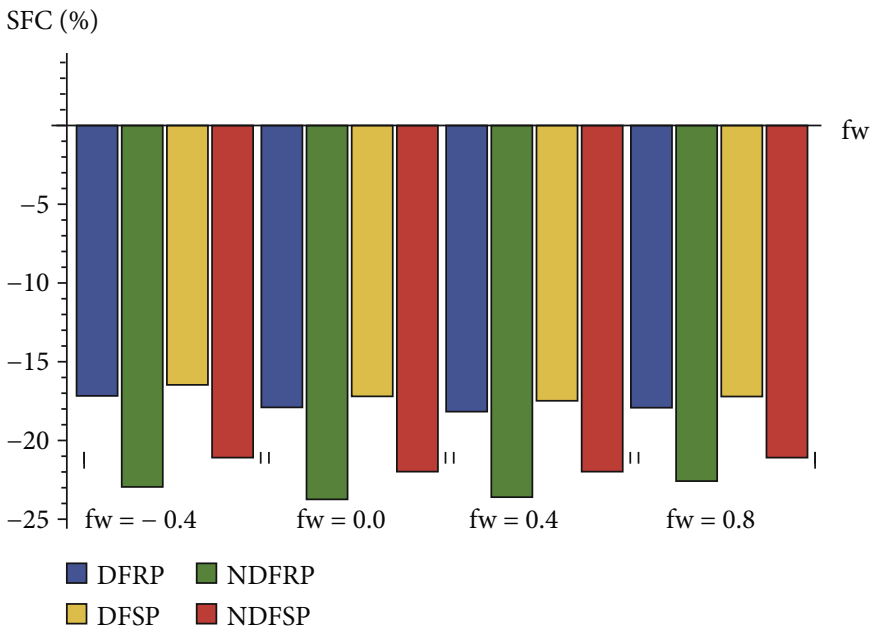

(b)

FiguRE 12: Decrement percentage of SFC for various values of fw on (a) Williamson fluid and (b) viscous fluid. 


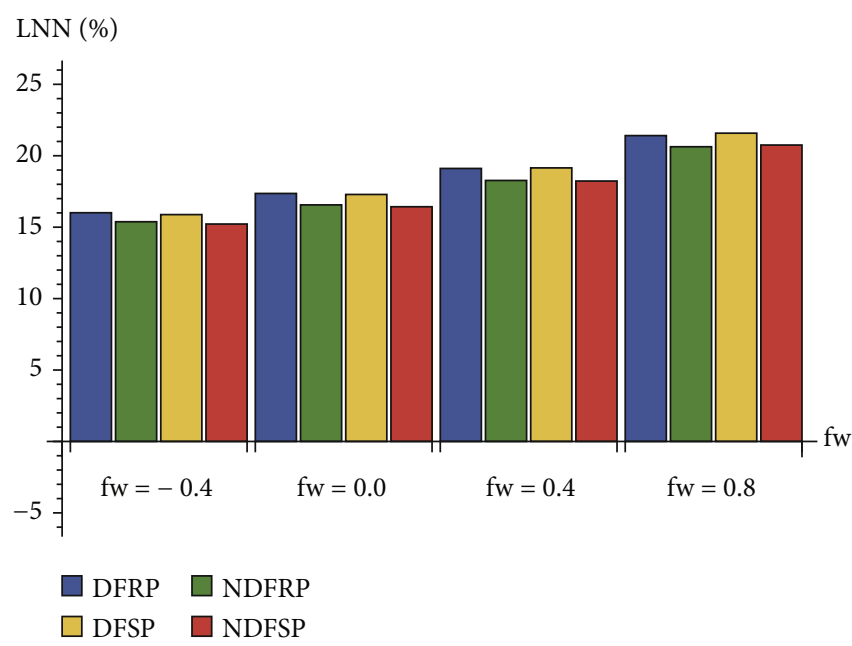

(a)

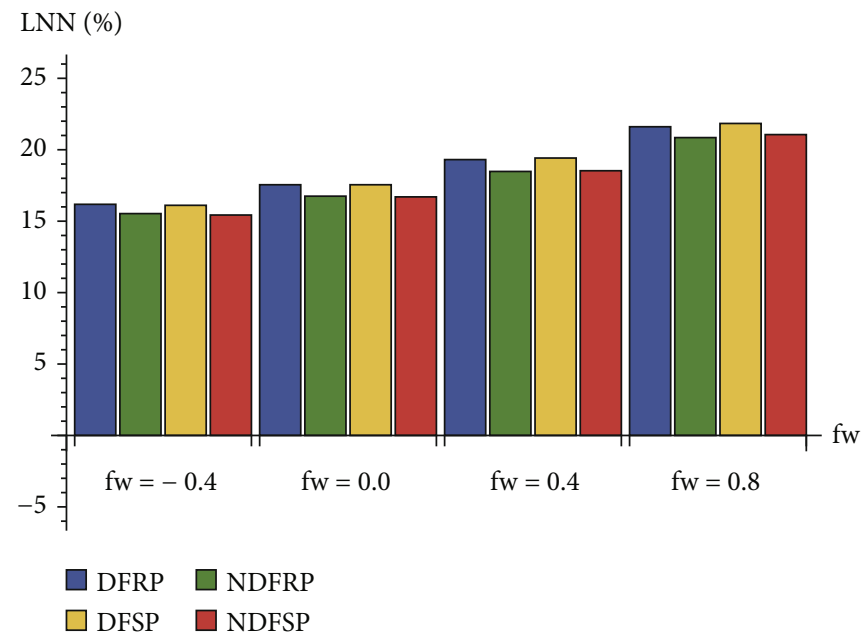

(b)

FIGURE 13: Increment percentage of LNN for various values of fw on (a) Williamson fluid and (b) viscous fluid.

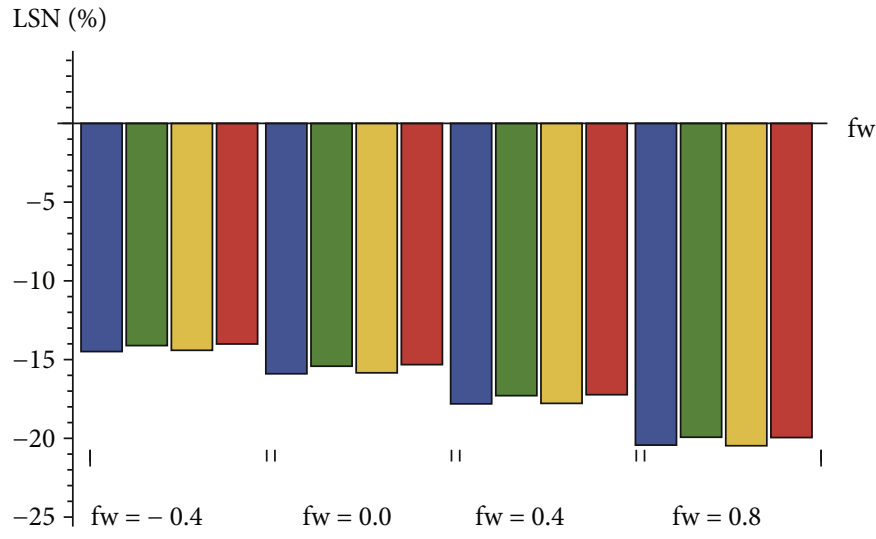

\section{$\square$ DFRP $\square$ NDFRP \\ $\square$ DFSP $\square$ NDFSP}

(a)

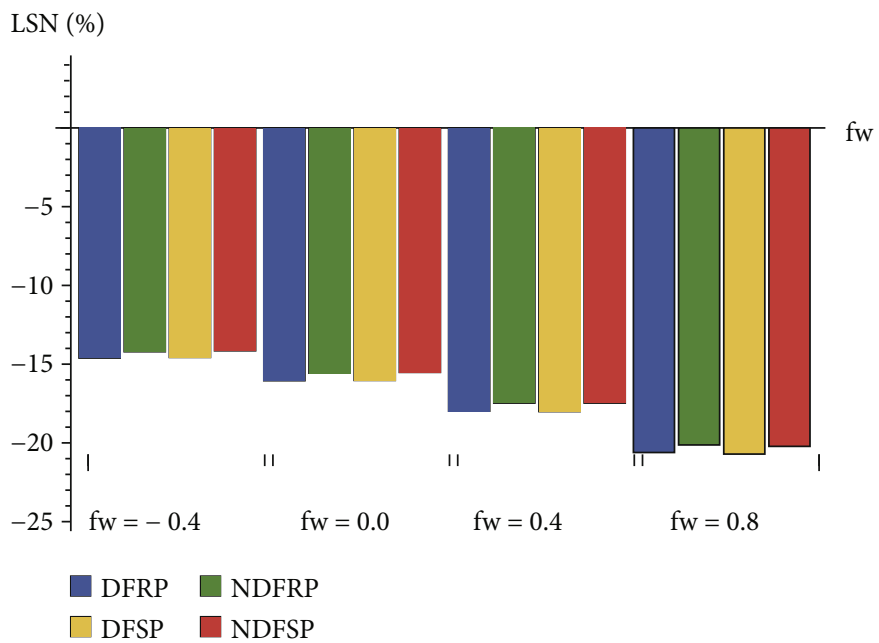

(b)

FIGURE 14: Decrement percentage of LSN for various values of fw on (a) Williamson fluid and (b) viscous fluid. 


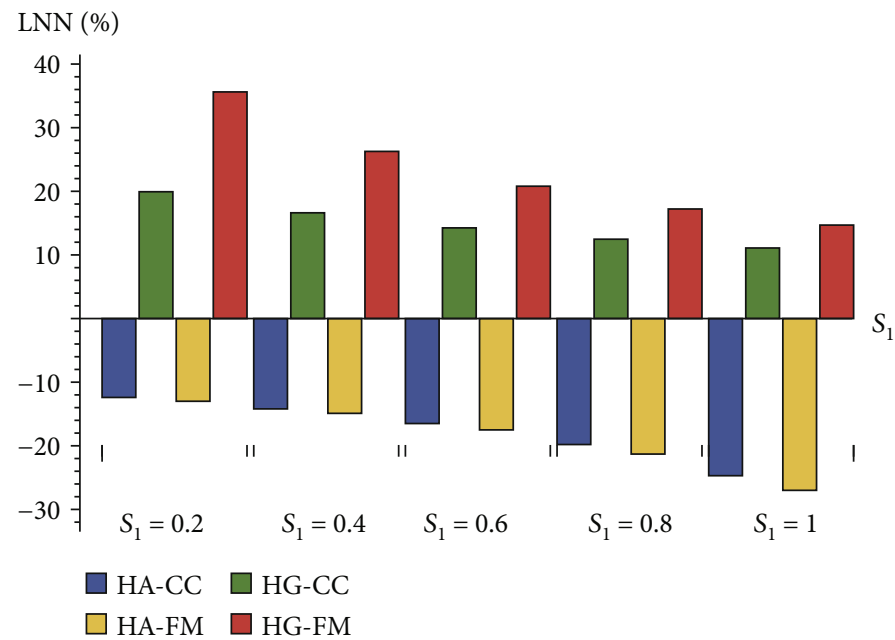

(a)

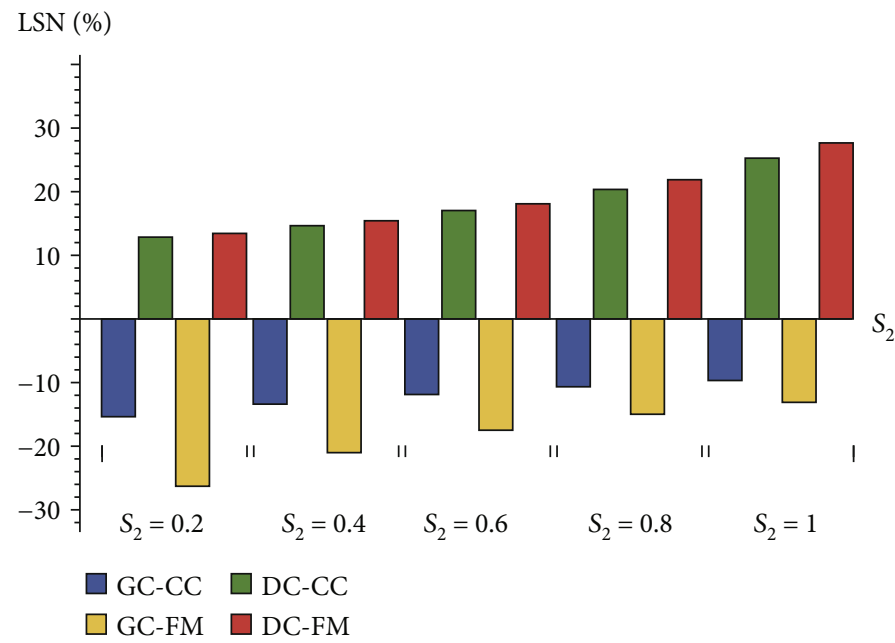

(b)

FIGURE 15: Decrement/increment percentage of (a) LNN for various values of $S_{1}$ and (b) LSN for various values of $S_{2}$.

for various values of $S_{1}$ and LSN for various values of $S_{2}$. We concluded that the heat transfer gradient enhances in heat generation case and maximum increment percentage is obtained when $S_{1}$ varies from 0 to 0.2 and it suppresses in heat consumption case and maximum decrement percentage is obtained when $S_{1}$ varies from 0.8 to 1 . The mass transfer gradient enhances in destructive chemical reaction case and maximum increment percentage is obtained when $S_{2}$ varies from 0.8 to 1 , and it suppresses in the generative case and maximum decrement percentage is obtained when $S_{2}$ varies from 0 to 0.2 .

\section{Conclusion}

The current study figures out the Darcy-Forchheimer flow of the Williamson fluid over a Riga plate with CattaneoChristov double diffusion and double stratification. The solutions are fetched numerically by a bvp4c solver in MATLAB and analytically by HAM. The outcomes are employed as follows: (i) The fluid velocity drops when the suction/injection parameter enriches

(ii) The larger values of the thermal radiation parameter boost up the temperature

(iii) The high temperature is noticed in the heat generation case, and the low temperature is produced in the heat absorption case

(iv) The generative chemical reaction case upturns the fluid concentration while the destructive chemical reaction case declines

(v) The high wall shear stress is produced when enlarging the modified Hartmann number
Abbreviations
CC: Cattaneo-Christov
DC: Destructive chemical reaction
DF: Darcy-Forchheimer 
FM: $\quad$ Fourier model

GC: Generative chemical reaction

HA: Heat absorption

HG: Heat generation

LNN: Local Nusselt number

LSN: Local Sherwood number

MBLT: Momentum boundary layer thickness

NDF: Non-Darcy-Forchheimer

RP: $\quad$ Riga plate

SFC: $\quad$ Skin friction coefficient

SP: $\quad$ Stationary plate.

\section{Data Availability}

The raw data supporting the conclusions of this article will be made available by the authors without undue reservation.

\section{Conflicts of Interest}

The authors declare that they have no competing interests.

\section{Authors' Contributions}

All authors contributed equally to this work. And all the authors have read and approved the final version of the manuscript.

\section{Acknowledgments}

This research was funded by the Deanship of Scientific Research at Princess Nourah Bint Abdulrahman University through the Fast-track Research Funding Program.

\section{References}

[1] R. V. Williamson, "The flow of pseudoplastic materials," Industrial and Engineering Chemistry Research, vol. 21, no. 11, pp. 1108-1111, 1929.

[2] M. R. Krishnamurthy, B. C. Prasannakumara, B. J. Gireesha, and R. S. R. Gorla, "Effect of chemical reaction on MHD boundary layer flow and melting heat transfer of Williamson nanofluid in porous medium," Engineering Science and Technology, An International Journal, vol. 19, no. 1, pp. 53-61, 2016.

[3] M. I. Khan, F. Alzahrani, A. Hobiny, and Z. Ali, "Modeling of Cattaneo-Christov double diffusions (CCDD) in Williamson nanomaterial slip flow subject to porous medium," Journal of Materials Research and Technology, vol. 9, no. 3, pp. 61726177, 2020.

[4] T. Hayat, A. Shafiq, and A. Alsaedi, "Hydromagnetic boundary layer flow of Williamson fluid in the presence of thermal radiation and Ohmic dissipation," Alexandria Engineering Journal, vol. 55, no. 3, pp. 2229-2240, 2016.

[5] S. Nadeem, S. T. Hussain, and C. Lee, "Flow of a Williamson fluid over a stretching sheet," Brazilian Journal of Chemical Engineering, vol. 30, no. 3, pp. 619-625, 2013.

[6] T. Salahuddin, M. Y. Malik, H. Arif, S. Bilal, and M. Awais, "MHD flow of Cattanneo-Christov heat flux model for Williamson fluid over a stretching sheet with variable thickness: using numerical approach," Journal of Magnetism and Magnetic Materials, vol. 401, no. 1, pp. 991-997, 2016.
[7] N. A. Khan and H. Khan, "A boundary layer ows of nonNewtonian Williamson fluid," Nonlinear Engineering, vol. 3, no. 2, pp. 107-115, 2014.

[8] T. Kebede, E. Haile, G. Awgichew, and T. Walelign, "Heat and mass transfer in unsteady boundary layer flow of Williamson nanouids," Journal of Applied Mathematics, vol. 2020, Article ID 1890972, 13 pages, 2020.

[9] P. Forchheimer, "Wasserbewegung durch boden," Zeitschrift des Vereines Deutscher Ingenieure, vol. 45, pp. 1782-1788, 1901.

[10] S. Mukhopadhyay, P. R. De, K. Bhattacharyya, and G. C. Layek, "Forced convective flow and heat transfer over a porous plate in a Darcy-Forchheimer porous medium in presence of radiation," Meccanica, vol. 47, no. 1, pp. 153-161, 2011.

[11] T. Hayat, A. Aziz, T. Muhammad, and A. Alsaedi, "Darcy-Forchheimer three-dimensional flow of Williamson nanofluid over a convectively heated nonlinear stretching surface," Communications in Theoretical Physics, vol. 68, no. 3, pp. 387-394, 2017.

[12] M. I. Khan, T. Hayat, and A. Alsaedi, "Numerical analysis for Darcy-Forchheimer flow in presence of homogeneous- heterogeneous reactions," Results in Physics, vol. 7, pp. 2644-2650, 2017.

[13] F. Haider, T. Hayat, and A. Alsaedi, "Flow of hybrid nanofluid through Darcy-Forchheimer porous space with variable characteristics," Alexandria Engineering Journal, vol. 60, no. 3, pp. 3047-3056, 2021.

[14] M. A. Sadiq, F. Haider, T. Hayat, and A. Alsaedi, "Partial slip in Darcy-Forchheimer carbon nanotubes flow by rotating disk," International Communications in Heat and Mass Transfer, vol. 116, p. 104641, 2020.

[15] T. Muhammad, K. Rafique, M. Asma, and M. Alghamdi, "Darcy-Forchheimer flow over an exponentially stretching curved surface with Cattaneo-Christov double diffusion," Physica A, vol. 556, article 123968, 2020.

[16] M. K. Nayak, S. Shaw, M. Ijaz Khan, V. S. Pandey, and M. Nazeer, "Flow and thermal analysis on DarcyForchheimer flow of copper-water nanofluid due to a rotating disk: a static and dynamic approach," Journal of Materials Research and Technology, vol. 9, no. 4, pp. 7387-7408, 2020.

[17] K. Loganathan, N. Alessa, K. Tamilvanan, and F. S. Alshammari, "Significances of Darcy-Forchheimer porous medium in third-grade nanofluid flow with entropy features," The European Physical Journal Special Topics, vol. 230, no. 5, pp. 1293-1305, 2021.

[18] J. C. Umavathi, O. Ojjela, and K. Vajravelu, "Numerical analysis of natural convective flow and heat transfer of nanofluids in a vertical rectangular duct using Darcy-ForchheimerBrinkman model," International Journal of Thermal Sciences, vol. 111, pp. 511-524, 2017.

[19] A. Gailitis and O. Lielausis, "On a possibility to reduce the hydrodynamic resistance of a plate in an electrolyte," Applied Magnetohydrodynamics, Reports of the Physics Institute, vol. 12, no. 1, pp. 143-146, 1961.

[20] A. Ahmad, S. Asghar, and S. Afzal, "Flow of nanofluid past a Riga plate," Journal of Magnetism and Magnetic Materials, vol. 402, pp. 44-48, 2016.

[21] M. Nazeer, M. I. Khan, M. U. Rafiq, and N. B. Khan, "Numerical and scale analysis of Eyring-Powell nanofluid towards a magnetized stretched Riga surface with entropy generation and internal resistance," International Communications in Heat and Mass Transfer, vol. 119, article 104968, 2020. 
[22] B. J. Gireesha, K. Ganesh Kumar, and B. C. Prasannakumar, "Scrutinization of chemical reaction effect on flow and mass transfer of Prandtl liquid over a Riga plate in the presence of solutal slip effect," International Journal of Chemical Reactor Engineering, vol. 16, no. 8, 2018.

[23] R. Mehmood, M. K. Nayak, N. S. Akber, and O. D. Makinde, "Effects of thermal-diffusion and diffusion-thermo on oblique stagnation point ow of couple stress Casson uid over a stretched horizontal Riga plate with higher-order chemical reaction," Journal of Nanouids, vol. 8, no. 1, pp. 94-102, 2019.

[24] M. Ayub, T. Abbas, and M. M. Bhatti, "Inspiration of slip effects on electromagnetohy-drodynamics (EMHD) nanouid ow through a horizontal Riga plate," European Physical Journal Plus, vol. 131, no. 93, pp. 1-9, 2016.

[25] N. K. Nayak, S. Shaw, O. D. Makinde, and A. J. Chamkha, "Investigation of partial slip and viscous dissipation effects on the radiative tangent hyperbolic nanouid flow past a vertical permeable Riga plate with internal heating: Bungiorno model," Journal of Nanouids, vol. 8, pp. 1-12, 2019.

[26] G. Rasool, T. Zhang, and A. Shafiq, "Second grade nanofluidic flow past a convectively heated vertical Riga plate," Physica Scripta, vol. 94, no. 12, p. 125212, 2019.

[27] C. Y. Cheng, "Combined heat and mass transfer in natural convection flow from a vertical wavy surface in a power-law fluid saturated porous medium with thermal and mass stratification," International Communications in Heat and Mass Transfer, vol. 36, no. 4, pp. 351-356, 2009.

[28] M. Khan, A. Rasheed, T. Salahuddin, and S. Ali, "Chemically reactive flow of hyperbolic tangent fluid flow having thermal radiation and double stratification embedded in porous medium," Ain Shams Engineering Journal, 2021.

[29] K. U. Rehman, A. A. Khan, M. Y. Malik, U. Ali, and M. Naseer, "Numerical analysis subject to double stratification and chemically reactive species on Williamson dual convection fluid flow yield by an inclined stretching cylindrical surface," Chinese Journal of Physics, vol. 55, no. 4, pp. 1637-1652, 2017.

[30] M. Khan, T. Salahuddin, M. Y. Malik, and F. O. Mallawi, "Change in viscosity of Williamson nanofluid flow due to thermal and solutal stratification," International Journal of Heat and Mass Transfer, vol. 126, pp. 941-948, 2018.

[31] O. M. Mallawi, M. Bhuvaneswari, S. Sivasankaran, and S. Eswaramoorthi, "Impact of double-stratification on convective flow of a non-Newtonian liquid in a Riga plate with Cattaneo-Christov double-flux and thermal radiation," Ain Shams Engineering Journal, vol. 12, no. 1, pp. 969-981, 2021.

[32] T. Hayat, M. Imtiaz, and A. Alsaedi, "Unsteady flow of nanofluid with double stratification and magnetohydrodynamics," International Journal of Heat and Mass Transfer, vol. 92, pp. 100-109, 2016.

[33] S. Sarwar and M. M. Rashidi, "Approximate solution of twoterm fractional-order diffusion, wave-diffusion, and telegraph models arising in mathematical physics using optimal homotopy asymptotic method," Waves in Random and Complex Media, vol. 26, no. 3, pp. 365-382, 2016.

[34] S. Eswaramoorthi, M. Bhuvaneswari, S. Sivasankaran, and S. Rajan, "Soret and Dufour effects on viscoelastic boundary layer flow over a stretching surface with convective boundary condition with radiation and chemical reaction," Scientia Iranica B, vol. 23, no. 6, pp. 2575-2586, 2016.
[35] H. Hassan and M. Mehdi Rashidi, "An analytic solution of micropolar flow in a porous channel with mass injection using homotopy analysis method," International Journal of Numerical Methods for Heat \& Fluid Flow, vol. 24, no. 2, pp. 419-437, 2014.

[36] K. Loganathan, N. Alessa, N. Namgyel, and T. S. Karthik, "MHD flow of thermally radiative Maxwell fluid past a heated stretching sheet with Cattaneo-Christov dual diffusion," Journal of Mathematics, vol. 2021, Article ID 5562667, 10 pages, 2021.

[37] M. Bhuvaneswari, S. Eswaramoorthi, S. Sivasankaran, and A. S. Alshomrani, "Effect of viscous dissipation and convective heating on convection ow of a second grade fluid over a stretching surface: analytical and numerical study," Scientia Iranica B, vol. 26, no. 3, pp. 1350-1357, 2019. 